\title{
Gamma camera imaging for studying intestinal absorption and whole-body distribution of selenomethionine
}

\author{
Jan L. Madsen ${ }^{1 *}$, Katarina Sjögreen-Gleisner ${ }^{2}$, Dennis R. Elema ${ }^{3}$, Lasse R. Søndergaard ${ }^{1}$, \\ Palle Rasmussen $^{3}$, Stefan Fuglsang ${ }^{1}$, Michael Ljungberg ${ }^{2}$ and Morten Damgaard ${ }^{1}$ \\ ${ }^{1}$ Department of Clinical Physiology and Nuclear Medicine, Centre of Functional Imaging and Research, \\ Hvidovre Hospital, Kettegård Allé 30, DK-2650 Hvidovre, Denmark \\ ${ }^{2}$ Department of Medical Radiation Physics, Clinical Sciences, Lund University, Lund, Sweden \\ ${ }^{3}$ Hevesy Laboratory, Technical University of Denmark, DTU Nutech, Roskilde, Denmark
}

(Submitted 25 February 2013 - Final revision received 4 July 2013 - Accepted 5 July 2013 - First published online 9 August 2013)

\section{Abstract}

Se metabolism in humans is not well characterised. Currently, the estimates of Se absorption, whole-body retention and excretion are being obtained from balance and tracer studies. In the present study, we used gamma camera imaging to evaluate the whole-body retention and distribution of radiolabelled selenomethionine (SeMet), the predominant form of Se present in foods. A total of eight healthy young men participated in the study. After consumption of a meal containing $\left.4 \mathrm{MBq}\left[{ }^{75} \mathrm{Se}\right] \mathrm{L}-\mathrm{SeMet}\left({ }^{75} \mathrm{Se}\right] \mathrm{SeMet}\right)$, whole-body gamma camera scanning was performed for $45 \mathrm{~min}$ every hour over a $6 \mathrm{~h}$ period, every second hour for the next $18 \mathrm{~h}$ and once on each of the subsequent $6 \mathrm{~d}$. Blood, urine and faecal samples were collected to determine the plasma content of $\left[{ }^{75}\right.$ Se]SeMet as well as its excretion in urine and faeces. Imaging showed that 87.9 (SD 3.3) \% of the administered activity of $\left[{ }^{75}\right.$ Se]SeMet was retained within the body after $7 \mathrm{~d}$. In contrast, the measured excretion in urine and faeces for the $7 \mathrm{~d}$ period was 8.2 (SD 1.1) \% of the activity. Time-activity curves were generated for the whole body, stomach, liver, abdomen (other than the stomach and the liver), brain and femoral muscles. Gamma camera imaging allows for the assessment of the postprandial absorption of SeMet. This technique may also permit concurrent studies of organ turnover of SeMet.

\section{Key words: Selenomethionine: Absorption: Whole-body distribution: Gamma camera imaging}

Se is recognised as a nutrient that is essential for human health $^{(1,2)}$. An inadequate Se supply seems to be associated with an increased risk of $\mathrm{CVD}^{(3,4)}$. Other studies have reported a relationship between low se exposure and risk of cancer $^{(5-7)}$. In foods and supplements, Se exists in a number of organic and inorganic forms including selenomethionine (SeMet), selenocysteine, selenate and selenite. The predominant form of Se in foods is organically bound, primarily in the form of SeMet ${ }^{(8,9)}$. Bioavailability and tissue distribution depend on the form ingested. Thus, SeMet is more effective in increasing the apparent Se status because it is non-specifically incorporated into proteins in place of methionine ${ }^{(10)}$. SeMet is a less-available metabolic source of Se compared with selenite and selenate, since these only need to be reduced to selenide to provide selenophosphate, the precursor of selenocysteine, the active form of Se in selenoproteins $^{(11)}$. Despite this, organic forms are often preferred in interventions, partly because they are less acutely toxic ${ }^{(8,9)}$.
Intestinal absorption of inorganic and organic Se compounds occurs via different routes and by different mechanisms. Membrane transport proteins with the capacity to mediate the uptake of organic forms of Se have been identified. Thus, studies have revealed that the selenoamino acids SeMet, selenocysteine and methylselenocysteine are transported effectively by a suite of amino acid transporters ${ }^{(12)}$. The incorporation of dietary Se into selenoproteins occurs through a series of interconvertions, many details of which are still lacking. Se metabolites are excreted in urine, faeces and breath mainly as selenosugars and methylated compounds ${ }^{(13)}$

Human studies on Se absorption and metabolism have been predominantly based on the recovery of ingested tracers in blood, urine and faeces ${ }^{(14,15)}$. A simple, direct method for assessing Se absorption and turnover is not available. Gamma camera imaging is currently being used to follow the organ uptake of a variety of radiolabelled tracers for diagnostic purposes. Our hypothesis was that the gamma camera

Abbreviations: $\left[{ }^{75}\right.$ Se $]$ SeMet, $\left[{ }^{75} \text { Se }\right]_{L}-$ Selenomethionine; SeMet, selenomethionine

*Corresponding author: Dr J. L. Madsen, fax +453862 3750, email jan.lysgaard.madsen@regionh.dk 
technique might as well constitute a useful imaging method for following the absorption and turnover of SeMet. Therefore, the objectives of the present study were to assess whether gamma camera imaging after oral intake of radiolabelled SeMet can be used to quantify the gastrointestinal absorption capacity of SeMet and to follow its postprandial distribution within the body.

\section{Materials and methods}

A total of eight healthy men (age 25 (SD 3) years, weight $77 \cdot 2$ (SD 5.3) kg, height $1.82(\mathrm{SD} 0.04) \mathrm{m}$, BMI $23.2(\mathrm{SD} 1.4) \mathrm{kg} / \mathrm{m}^{2}$ and plasma volume 3.31 (SD $0 \cdot 16$ ) litres) participated in the study. All participating men had normal plasma Se levels before the study $(1 \cdot 11(\mathrm{sD} 0 \cdot 11) \mu \mathrm{mol} / \mathrm{l})$. None of the participants had undergone abdominal surgery previously (other than an appendectomy) or taken any medication. The present study was conducted according to the guidelines laid down in the Declaration of Helsinki, and all procedures involving human subjects were approved by the Scientific Ethics Committees of the Capital Region of Denmark (protocol no. H-3-2009-092) and the Danish Data Protection Agency (journal no. 2009-41-3751). Written informed consent was obtained from all the participants.

\section{Procedure}

After at least a $10 \mathrm{~h}$ fast, a cannula was inserted into the cubital vein of the participants at 08.00 hours for blood sampling. Then, whole-body transmission scanning was performed. After having voided and discarded the urine, the participants ingested a $\left.\left.{ }^{75} \mathrm{Se}\right]_{\mathrm{L}-S e M e t}\left({ }^{75} \mathrm{Se}\right] \mathrm{SeMet}\right)$-containing meal between 08.45 and 09.00 hours. Immediately after consumption of the meal, a whole-body emission scanning was performed. The procedure was repeated every hour over a $6 \mathrm{~h}$ period, every second hour for the next $18 \mathrm{~h}$ and once at 09.00 hours on each of the subsequent $6 \mathrm{~d}$. A total of twenty-two emission scans were acquired from each participant.

After each emission scanning session, a $10 \mathrm{ml}$ blood sample was drawn to follow the plasma concentration of ${ }^{75} \mathrm{Se}$. Starting at 09.00 hours on the 1 st day, urine was collected at $2 \mathrm{~h}$ periods until 09.00 hours the next morning. Then, urine was collected at $24 \mathrm{~h}$ periods for the next $6 \mathrm{~d}$. Starting at 09.00 hours on the 1 st day, faeces were sampled at $24 \mathrm{~h}$ periods for the next $7 \mathrm{~d}$.

\section{$\Gamma^{75}$ Se $J$-selenomethionine}

$\left[{ }^{75}\right.$ Se]SeMet was produced and delivered by the Hevesy Laboratory, DTU Nutech. The radionuclide, ${ }^{75} \mathrm{Se}$, was produced by neutron irradiation of $5 \mathrm{mg}$ of natural Se metal. Neutron irradiation for 4 weeks at the University of Missouri Research Reactor Center, Columbia, MI, USA, yielded $687 \mathrm{MBq}{ }^{75} \mathrm{Se}$ as measured at the start of ${ }^{75}$ Se]SeMet synthesis, as described previously ${ }^{(16)}$. A fraction of $\left[{ }^{75}\right.$ Se]SeMet obtained from the synthesis was purified using preparative HPLC with an aqueous $0.9 \% \mathrm{NaCl}$ solution as the eluent. After dilution and sterile filtration, the final formulation contained $92 \mathrm{MBq}$ $\left[{ }^{75}\right.$ Se]SeMet in $30 \mathrm{ml}$ of aqueous $0.9 \% \mathrm{NaCl}$. The specific activity was $10.9 \mathrm{GBq} / \mathrm{mmol}$. The radionuclidic purity of the product was measured with germanium detector spectroscopy, and no impurities were detected. The radiochemical purity was determined to be $>98 \%$ by analytical HPLC.

\section{$\left.{ }^{75} \mathrm{Se}\right]$ L-selenomethionine-containing meal}

The $\left[{ }^{75}\right.$ Se]SeMet-containing meal comprised $2970 \mathrm{~kJ}$ (16\% protein, $28 \%$ fat and $56 \%$ carbohydrates) and consisted of black bread, butter, chicken, cheese, jam, yogurt, muesli and $350 \mathrm{ml}$ of water with $4 \mathrm{MBq}\left[{ }^{75}\right.$ Se]SeMet comprising $29 \cdot 0 \mu \mathrm{g}$ Se (of which only $0.027 \%$ was radioactive).

\section{$24 \mathrm{~h}$ diet regimen}

Additional meals comprising $11816 \mathrm{~kJ}$ were ingested after emission scanning at 12.45, 16.45, 18.45, 20.45, 22.45, 02.45 and 06.45 hours. Because of the imaging procedures, blood sampling and urine sample collection, the participants were awake for longer periods of the night. Therefore, we found that it was most convenient for the participants if three small meals were served during the night. Every second hour, the participants drank $350 \mathrm{ml}$ of water.

\section{Imaging system}

Imaging was performed using a dual-head gamma camera equipped with a single-slice computed tomography system (Infinia VC HawkEye; General Electric Medical Systems) and connected to a dedicated image processing system (Xeleris; General Electric Medical Systems).

\section{Transmission scanning}

For the purpose of subject-specific attenuation correction, a whole-body planar transmission image (X-ray scout image) was acquired using the X-ray unit of the imaging system. The image matrix had a size of $384 \times 2000$ pixels, each with a size of $1 \mathrm{~mm}^{2}$, and the total scanning time was approximately $2 \mathrm{~min}$. For the determination of an attenuation correction map for ${ }^{75} \mathrm{Se}$, valid for the gamma photons detected in the emission scanning windows, linear attenuation coefficients for soft tissue and bone were first calculated from the reference data $^{(17)}$. Abundance-weighted averages of the attenuation coefficient values for 136,265 and $280 \mathrm{keV}$ were calculated for soft tissue and bone. The pixel values in the acquired scout image were then rescaled by multiplication to a factor determined from the weight of the participants, by assuming a representative photon energy of $70 \mathrm{keV}$ for the X-ray bremsstrahlung spectrum and by performing a scaling from the linear attenuation values of $70 \mathrm{keV}$ to the photon energies used for ${ }^{75}$ Se imaging ${ }^{(18)}$. 
(a)
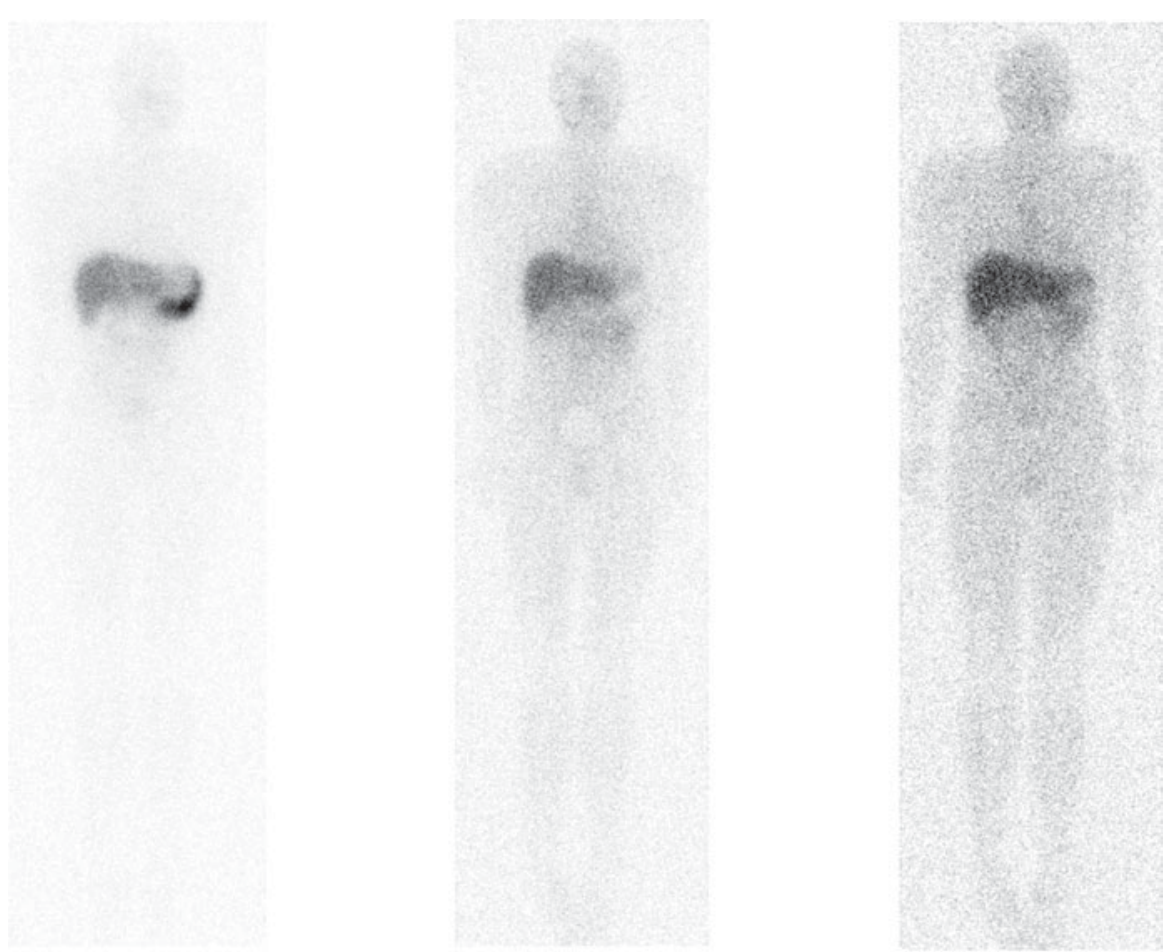

(b)

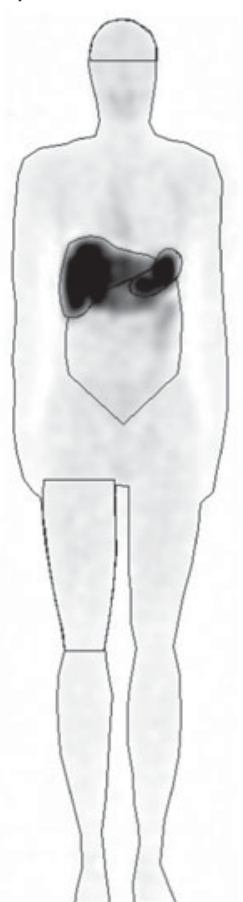

$1 \mathrm{~h}$

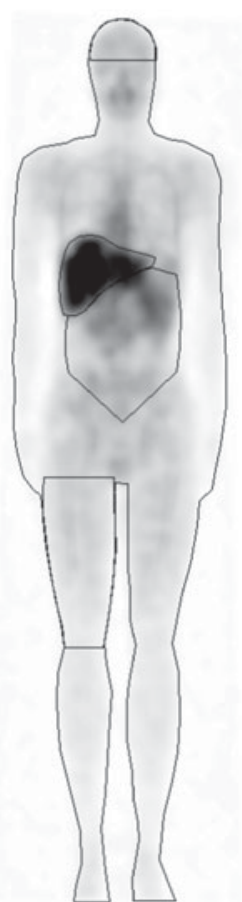

$24 \mathrm{~h}$

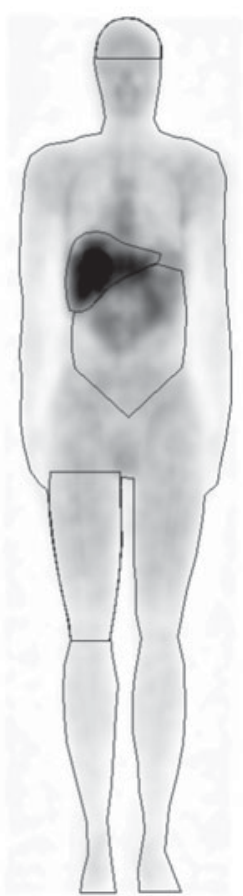

$168 \mathrm{~h}$

Fig. 1. Examples of emission (gamma camera) images after oral ingestion of $\left[{ }^{75} \mathrm{Se}\right] \mathrm{L}-\mathrm{selenomethionine.} \mathrm{(a)} \mathrm{Raw} \mathrm{non-corrected} \mathrm{images.} \mathrm{(b)} \mathrm{Images} \mathrm{corrected} \mathrm{for}$ scattered gamma radiation, septal penetration of gamma radiation, and gamma ray attenuation and with the various regions of interest (whole body, stomach, liver, abdomen other than the stomach and the liver, brain and femoral muscles) superposed. 


\section{Emission scanning}

The distribution of ${ }^{75} \mathrm{Se}$ was investigated by planar wholebody emission scanning. Anterior and posterior projections were acquired from the participants in the supine position. Medium-energy, all-purpose collimators and $136 \pm 10 \%$ and $272 \pm 12 \cdot 5 \% \mathrm{keV}$ energy windows were used. The principal gamma emissions in the ${ }^{75} \mathrm{Se}$ decay that were detected in these energy windows were 136, 265 and $280 \mathrm{keV}$ with emission abundances of 0.6059, 0.5940 and 0.2519, respectively. Whole-body images, composed of $256 \times 1024$ pixels, each measuring $5.76 \mathrm{~mm}^{2}$, were acquired at a scanning speed of $5 \mathrm{~cm} / \mathrm{min}$, implying a total scanning time of $45 \mathrm{~min}$.

\section{Image correction for scatter, septal penetration and photon attenuation}

For reliable quantification of ${ }^{75} \mathrm{Se}$ distribution, planar wholebody emission images were compensated for physical decay, scatter contribution, septal penetration and photon attenuation. In brief, the raw anterior-posterior images were corrected for scatter and septal penetration by means of a deconvolution procedure, in which the scatter point-spread functions were determined by Monte Carlo simulation for the specific camera system, radionuclide and average thickness of the participants over the abdominal region ${ }^{(19)}$. From the obtained primary count rate images, geometric mean images were calculated by pixel-by-pixel multiplication of the anterior and mirrored posterior images. Since the participants were allowed to move between transmission and emission scanning, co-registration of the emission images was necessary to enable pixel-based activity quantification. For this purpose, an automatic co-registration method was applied $^{(20)}$, in which the $5 \mathrm{~h}$ emission image was first co-registered to the attenuation correction map and the remaining emission images were registered to the co-registered $5 \mathrm{~h}$ image. The $5 \mathrm{~h}$ image was chosen as a reference since the activity had by then distributed within the body and thus exhibited characteristics favourable for registration to the attenuation correction map. Correction for photon attenuation was performed by pixel-by-pixel multiplication of the registered couples of geometric mean images and the attenuation map $^{(18)}$. The scatter-, septal penetration- and attenuation-compensated images were converted from counts/s to activity using a measured value of the system sensitivity (counts/s per MBq). This value was determined by imaging a ${ }^{75} \mathrm{Se}$ source located $10 \mathrm{~cm}$ from the surface of the collimator, using the collimator and energy windows used for emission scanning ${ }^{(19)}$. Finally, the obtained activity projection images were analysed for activity in different structures using twodimensional regions of interest that were delineated manually by the same observer in all the images.

\section{Plasma, urine and faecal sample analyses}

All blood samples were immediately centrifuged for $10 \mathrm{~min}$ at $1000 \mathrm{~g}$, and plasma was stored until analysis. On completion of the study, the weight of each urine and faecal portion was recorded. The faecal portions were homogenised with known amounts of water, and aliquots of urine and faeces were stored for later analysis. For the measurement of ${ }^{75} \mathrm{Se}$ activity, $3 \mathrm{ml}$ aliquots of plasma, urine and homogenised faecal samples and an appropriate dilution of the stock solution of $\left[{ }^{75}\right.$ Se]SeMet were counted for $30 \mathrm{~min}$ in a gamma well counter (Wizard 1480; Wallac). For conversion into counts for total plasma volume, the total plasma volume of each participant was estimated from height and weight data according to tabled references ${ }^{(21)}$. All counts were corrected for physical decay, converted into $\mathrm{Bq}$ and expressed as the percentage of the administered activity.

\section{Statistical analysis}

The whole-body loss of ${ }^{75}$ Se activity after $7 \mathrm{~d}$, as estimated from image data, and the measured excretion of ${ }^{75}$ Se activity in urine and faeces after $7 \mathrm{~d}$ were compared using the paired $t$ test. A $P$ value $<0.05$ was considered significant.

\section{Dosimetry}

Each participant received an effective absorbed dose of $10.6 \mathrm{mSv}$, of which $0.2 \mathrm{mSv}$ was from transmission scanning and $10 \cdot 4 \mathrm{mSv}$ from $\left[{ }^{75} \mathrm{Se}\right] \mathrm{SeMet}$. In contrast, the normal background radiation in the UK results in a yearly average effective absorbed dose of $2-3 \mathrm{mSv}$ and a standard thorax X-ray computed tomography results in an effective absorbed dose of about $10 \mathrm{mSv}$.

\section{Results}

The examples of raw non-corrected emission (gamma camera) images and the corresponding corrected images with superposed regions of interest are shown in Fig. 1. In all the participants, the accumulation of ${ }^{75} \mathrm{Se}$ activity permitted reliable delineation of the body contour, stomach and liver.

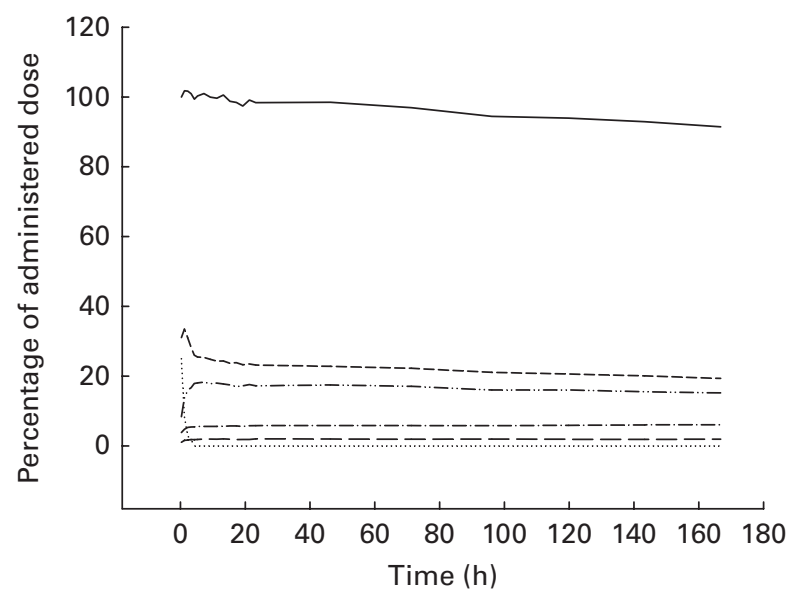

Fig. 2. Example of time-activity curves derived from the various regions of interest drawn on the corrected emission (gamma camera) images after oral ingestion of $\left[{ }^{75} \mathrm{Se}\right] \mathrm{L}$-selenomethionine. _ - Whole body; ......., stomach; --- , liver; ---, abdomen other than the stomach and the liver; -- , brain; -.-, femoral muscles. 


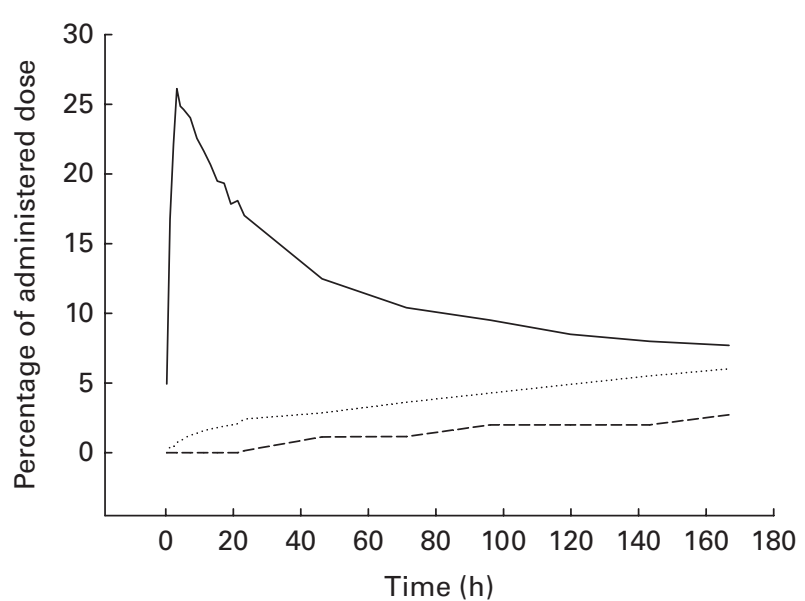

Fig. 3. Example of time-activity curves derived from the analysis of plasma $(-)$, urine (......) and faecal (---) samples after oral ingestion of $\left[{ }^{75}\right.$ Se]L-selenomethionine.

The resolution of the images was so low that we could not delineate the spleen, pancreas, small intestine or colon in any of the images. In the images of some of the participants, we could see the kidneys, but only in the posterior view. The body contour judged the delineation of areas over the brain, femoral muscles and abdomen (other than the stomach and the liver). Fig. 2 shows the retention of ${ }^{75}$ Se activity in the various regions of interest $v$. time for one participant. For one representative participant, the total plasma activity of ${ }^{75} \mathrm{Se}$ as a function of time, as well as the accumulated excretion of ${ }^{75} \mathrm{Se}$ in urine and faeces $v$. time, is shown in Fig. 3. Fig. 4 shows the whole-body loss of ${ }^{75} \mathrm{Se}$ activity against time as estimated from the quantified emission (gamma camera) images, as well as the accumulated excretion of ${ }^{75} \mathrm{Se}$ in urine and faeces $v$. time, for one participant. After $7 \mathrm{~d}$, the imaging showed that 87.9 (SD 3.3) \% of the administered activity was retained within the body; that is, $12 \cdot 1$ (SD $3 \cdot 3$ ) \% of the activity was lost from the body. In contrast, the accumulated excretion in urine and faeces was 8.2 (SD 1.1$) \%$ of the administered activity after $7 \mathrm{~d}(P=0.008)$.

\section{Discussion}

In 1961, Blau ${ }^{(22)}$ introduced ${ }^{75}$ Se]SeMet as a radiolabelled agent for gamma camera imaging of the pancreas. This amino acid was selected for examination because of its high rate of incorporation into pancreatic enzymes after intravenous injection. During the following decade, $\left[{ }^{75}\right.$ Se]SeMet was used for the scintigraphic detection of pancreatic and parathyroid adenomas as well as lymphomas ${ }^{(23-25)}$. However, the $120 \mathrm{~d}$ half-life of ${ }^{75} \mathrm{Se}$ limited the acceptable administered activity and consequently the quality of the acquired images. As a result, the procedure was outdistanced by the new and much more efficient ultrasonographic and X-ray computed tomography methods. Since then, the imaging quality of the gamma camera has improved, and we found it, therefore, relevant to evaluate the performance of scintigraphy for recording the whole-body retention and distribution of oral $\left[{ }^{75} \mathrm{Se}\right] \mathrm{SeMet}$.
SeMet is absorbed rapidly and almost completely from the normal human intestine and is an important organic nutritional source of Se that is essential for human health ${ }^{(1,2)}$. Much of the Se in plants is present in small selenoamino acid compounds derived from SeMet and selenocysteine, whereas animal-derived food products seem to contain Se mainly as SeMet and selenocysteine in proteins ${ }^{(8,9)}$. SeMet can be a constituent part of general protein synthesis ${ }^{(26)}$. Through the intermediate metabolite selenide, Se from nutritional SeMet is excreted predominantly in the urine in the form of selenosugars ${ }^{(27)}$.

In a previous study carried out by Griffiths et al. ${ }^{(14)}$, wholebody counting and urinary and faecal losses were used to follow the whole-body retention of an oral dose of $\left.{ }^{75} \mathrm{Se}\right] \mathrm{SeMet}$ in four healthy women. After $7 \mathrm{~d}$, the estimated whole-body retention from the measured loss in urine and faeces was 90-92\%, whereas whole-body counting showed a slightly lower retention. Our findings are in accordance with these previous observations. In parallel with this, the present imaging technique showed a whole-body loss of $\left.{ }^{75} \mathrm{Se}\right] \mathrm{SeMet}$ on day 7 that was slightly but significantly $(P=0.008)$ higher than the detected accumulated loss in urine and faeces after $7 \mathrm{~d}$ (Fig. 4). The reason for this difference is not clear, but at least some of the discrepancy may have resulted from the fact that we did not measure the amount of ${ }^{75} \mathrm{Se}$ that was excreted in the breath. Besides, incomplete sampling of urine and faeces or imperfect image-based corrections for photon attenuation and scatter may be possible reasons. The peak ${ }^{75} \mathrm{Se}$ plasma concentration after $4-6 \mathrm{~h}$ in the present study is within the range found by Griffiths et al. ${ }^{(14)}$. Thus, gamma camera recording of the whole-body retention of $\left.{ }^{75} \mathrm{Se}\right] \mathrm{SeMet}$ seems reliable for evaluating the overall intestinal absorption of SeMet.

For all the participants, we could clearly delineate the body contour, stomach and liver in the corrected images (Fig. 1), and presumably, therefore, the corresponding time-activity

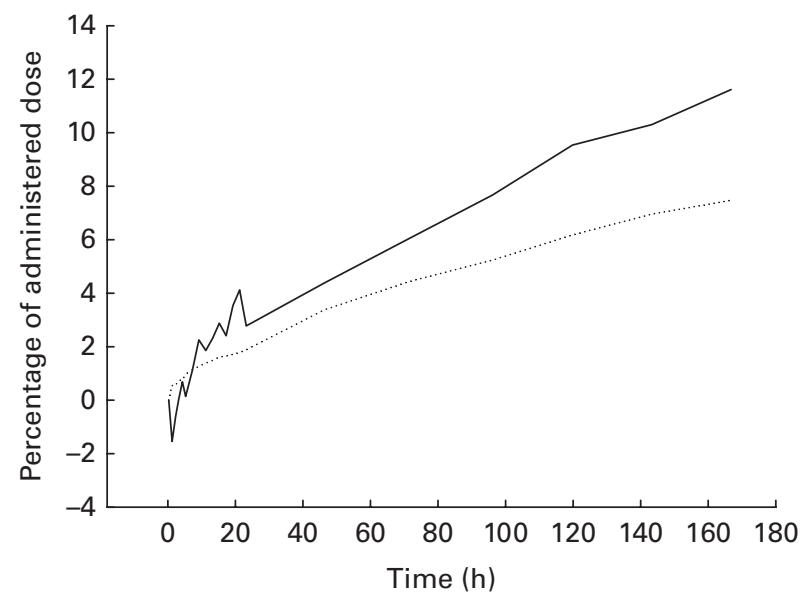

Fig. 4. Example of the time course of the whole-body loss (-) of ${ }^{75} \mathrm{Se}$ as estimated from the corrected emission (gamma camera) images and corresponding accumulated excretion of ${ }^{75} \mathrm{Se}$ in urine and faeces (.......) after oral ingestion of $\left[{ }^{75} \mathrm{Se}\right] \mathrm{L}$-selenomethionine. After $7 \mathrm{~d}$, the whole-body loss of ${ }^{75} \mathrm{Se}$ as found by imaging was significantly higher than the amount excreted in the urine and faeces in a group of eight healthy men $(P=0.008)$. 
curves (Figs. 2-4) are valid. Based on the reported concentrations of ${ }^{75}$ Se activity in tissue collected at surgery or autopsy after intravenous injection of $\left[{ }^{75} \mathrm{Se}\right] \mathrm{SeMet}$, the total liver content of ${ }^{75}$ Se seems to be $15-20 \%$ of the administered activity after $7 \mathrm{~d}^{(28,29)}$, a value that is in line with the present finding. The time-activity curves obtained for the liver probably reflect a complex interplay between the intestinal absorption of $\left[{ }^{75}\right.$ Se]SeMet, liver uptake and metabolism of $\left[{ }^{75} \mathrm{Se}\right]$ SeMet, and liver release of ${ }^{75} \mathrm{Se}$-containing compounds. In some clinical settings, therefore, a sequential gamma camera imaging procedure might prove useful for monitoring the aspects of parenchymatous liver function. Unless $\left[{ }^{75} \mathrm{Se}\right] \mathrm{SeMet}$ is absorbed from the stomach, which is unlikely, the time-activity curves for the stomach simply reflect gastric emptying of the tracer. Our data suggest a low uptake of SeMet, other Se-containing compounds or free Se in the brain and femoral muscles.

The present study has some limitations. First, the diet regimen aimed to reduce the effect of variations in meal composition and gastrointestinal motility on the absorption of $\left[{ }^{75} \mathrm{Se}\right]$ SeMet. For practical reasons, the regimen was followed only for the first $24 \mathrm{~h}$, and it is, therefore, conceivable that changing the intake of Se and SeMet during the rest of the study period increased the inter-subject variations in determined retention and excretion values. We did not measure the Se content of the initial meals and the plasma concentration of Se during the study. Possibly, inter-subject variations in Se status could explain some of the apparent differences in Se kinetics. However, the main objective of the study was to assess the overall efficacy of the imaging technique. Second, free amino acids can be absorbed via different amino acid transporters with certain group specificities in the small intestine ${ }^{(2)}$. Thus, normal whole-body retention of oral $\left[{ }^{75} \mathrm{Se}\right] \mathrm{SeMet}$ only rules out an impaired intestinal absorption of that specific amino acid in its free form. Third, as proteins and free amino acids behave differently at several digestive steps (such as gastric emptying and dietary protein hydrolysis and absorption), the time course of the appearance of a free amino acid in the plasma can be different from that of the same amino acid bound to proteins ${ }^{(30)}$. Consequently, the metabolic fate of free $\left[{ }^{75}\right.$ Se]SeMet does not necessarily reflect that of SeMet bound to dietary proteins. Fourth, quite arbitrarily, we only followed our participants for $7 \mathrm{~d}$. In all the participants, however, we observed a slow and almost linear decrease in liver/ abdominal ${ }^{75}$ Se levels after the initial peak. Therefore, it is not likely that imaging beyond $7 \mathrm{~d}$ would have added substantially to our observations. Lastly, our presentation would profit from compartmental modelling of the data, if possible. Thus, we hoped that the data could be used to confirm or improve some of the modelling-based variables found by Swanson et $a l .{ }^{(31)}$ and Wastney et $a l .{ }^{(15)}$. However, only the stomach and the liver were clearly depicted in all the images, leaving the data obtained for the liver only relevant for kinetic analysis. Furthermore, the time resolution of the initial part of the time-activity curves obtained for the liver was too low for proper use. Future dynamic studies with, for instance, 1 min image acquisitions of the abdominal region for a few hours together with simultaneous sampling of the plasma will probably yield information about the initial SeMet turnover that can be used for reliable compartmental modelling.

\section{Summary and perspectives}

Whole-body gamma camera imaging with appropriate correction for scatter, septal penetration and photon attenuation provides reliable recordings of the intestinal absorption of SeMet. Therefore, this method potentially provides the opportunity to non-invasively explore the effects of food composition, gastrointestinal motility, and gastrointestinal resection or bypass on the intestinal absorption of SeMet. Furthermore, the technique seems to allow postprandial recordings of the whole-body distribution of SeMet and thereby provides data that could be used to confirm or improve the existing models of human SeMet metabolism.

The overall strength of the gamma camera technique is its capability of tracing quantitatively small amounts of gamma ray-emitting substances in vivo. Thus, the technique allows the tracing of physiological amounts of radiolabelled nutrients or food elements other than SeMet. The weaknesses of the method are the restricted availability and exposure to radiation.

\section{Acknowledgements}

The authors thank Ingelise Siegumfeldt and Bente Pedersen for their assistance in plasma, urine and faecal sample analyses, and all the subjects for their participation in the study.

The present study was funded by grants from the Aase and Ejnar Danielsen Foundation (10-000243), the Hartmann Brothers Foundation (A7572), the P.A. Messerschmidt and Wife Foundation (028077-0002) and the Beckett Foundation (178PV/LS). All funders had no role in the design, analysis or writing of this article.

The authors' contributions are as follows: J. L. M. and M. D. designed the research; D. R. E. and P. R. prepared [ ${ }^{75}$ Se]SeMet; J. L. M., M. D., L. R. S. and S. F. conducted the research; J. L. M., K. S.-G., L. R. S., S. F. and M. L. analysed the data; J. L. M., K. S.-G., D. R. E. and M. L. wrote the paper; J. L. M. had primary responsibility for the final content. All authors read and approved the final manuscript. All authors declare no conflicts of interest.

\section{References}

1. Rayman PM (2000) The importance of selenium to human health. Lancet 356, 233-241.

2. Papp LV, Lu J, Holmgren A, et al. (2007) From selenium to selenoproteins: synthesis, identity, and their role in human health. Antioxid Redox Signal 9, 775-806.

3. Yang J, Wang T, Wu C, et al. (2010) Selenium level surveillance for the year 2007 of Keshan disease in endemic areas and analysis on surveillance results between 2003 and 2007. Biol Trace Elem Res 138, 53-59.

4. Frustaci A, Sabbioni E, Fortaner S, et al. (2012) Seleniumand zinc-deficient cardiomyopathy in human intestinal malabsorption: preliminary results of selenium/zinc infusion. Eur J Heart Fail 14, 202-210. 
5. Reid ME, Duffield-Lillico AJ, Sunga A, et al. (2006) Selenium supplementation and colorectal adenomas: an analysis of the nutritional prevention of cancer trial. Int $J$ Cancer 118, $1777-1781$

6. Dziaman T, Huzarski T, Gackowski D, et al. (2009) Selenium supplementation reduced oxidative DNA damage in adnexectomized BRCA1 mutations carriers. Cancer Epidemiol Biomarkers Prev 18, 2923-2928.

7. Hu Y, McIntosh GH \& Young GP (2012) Selenium-rich foods: a promising approach to colorectal cancer prevention. Curr Pharm Biotechnol 13, 165-172.

8. Schrauzer GN (2000) Selenomethionine: a review of its nutritional significance, metabolism and toxicity. $J$ Nutr 130, 1653-1656.

9. Schrauzer GN (2001) Nutritional selenium supplements: product types, quality, and safety. J Am Coll Nutr 20, 1-4.

10. Thomson CD, Robinson MF, Butler JA, et al. (1993) Longterm supplementation with selenate and selenomethionine: selenium and glutathione peroxidase (EC 1.11.1.9) in blood components of New Zealand women. Br J Nutr 69, 577-588.

11. Allan CB, Lacourciere GM \& Stadtman TC (1999) Responsiveness of selenoproteins to dietary selenium. Annu Rev Nutr 19, $1-16$

12. Nickel A, Kottra G, Schmidt G, et al. (2009) Characteristics of transport of selenoamino acids by epithelial amino acid transporters. Chem Biol Interact 177, 234-241.

13. Fairweather-Tait SJ, Bao Y, Broadley MR, et al. (2011) Selenium in human health and disease. Antioxid Redox Signal 14, 1337-1383.

14. Griffiths NM, Stewart RDH \& Robinson MF (1976) The metabolism of $\left[{ }^{75}\right.$ Se]selenomethionine in four women. $\mathrm{BrJ}$ Nutr 35, 373-382.

15. Wastney ME, Combs GF Jr, Canfield WK, et al. (2011) A human model of selenium that integrates metabolism from selenite and selenomethionine. J Nutr 141, 708-717.

16. Plenevaux A, Cantineau R, Guillaume M, et al. (1987) Fast chemical synthesis of $\left[{ }^{75} \mathrm{Se}\right] \mathrm{L}-\mathrm{selenomethionine.} \mathrm{Appl} \mathrm{Radiat}$ Isot 38, 59-61.

17. Hubbell JH, Coursey JS, Hwang J, et al. (2003) Bibliography of Photon Total Cross Section (Attenuation Coefficient) Measurements (Version 2.3). National Institute of Standards and Technology. www.nist.gov/pml/data/photon_cs (accessed May 2012).
18. Gleisner KS \& Ljungberg M (2012) Patient-specific whole-body attenuation correction maps from a CT system for conjugate-view-based activity quantification: method development and evaluation. Cancer Biother Radiopharm 27, 652-664.

19. Sjogreen K, Ljungberg M \& Strand S-E (2002) An activity quantification method based on registration of CT and whole-body scintillation gamma cameras, with application to 131I. J Nucl Med 43, 972-982.

20. Sjogreen K, Ljungberg M, Wingardh K, et al. (2001) Registration of emission and transmission whole-body scintillation-camera images. J Nucl Med 42, 1563-1570.

21. Hurley PJ (1975) Red cell and plasma volumes in normal adults. J Nucl Med 16, 46-52.

22. Blau M (1961) Biosynthesis of ${ }^{75}$ Se-selenomethionine and ${ }^{75}$ Se-selenocystine. Biochem Biophys Acta 49, 389-390.

23. Blau M \& Bender MA (1962) Se ${ }^{75}$-selenomethionine for visualization of the pancreas by isotope scanning. Radiology $\mathbf{7 8}, 974$.

24. McGeown MG, Bell TK, Soyannwo MA, et al. (1968) Parathyroid scanning in the human with selenomethionine- ${ }^{75}$ Se. Br J Radiol 41, 300-306.

25. Herrera NE, Gonzales R, Schwartz RD, et al. (1965) ${ }^{75} \mathrm{Se}$ methionine as a diagnostic agent in malignant lymphoma. J Nucl Med 6, 792-804.

26. Hawkes WC, Alkan FZ \& Oehler L (2003) Absorption, distribution and excretion of selenium from beef and rice in healthy North American men. J Nutr 133, 3434-3442.

27. Suzuki KT, Kurasaki K, Okazaki N, et al. (2005) Selenosugar and trimethylselenium among urinary Se metabolites: doseand age-related changes. Toxicol Appl Pharmacol 206, 1-8.

28. Ben-Porath M \& Kaplan E (1969) The distribution and concentration of ${ }^{75} \mathrm{Se}-$ selenomethionine in man. J Nucl Med 10, 709-710.

29. Lathrop KA, Johnston RE, Blau M, et al. (1972) Radiation dose to humans from ${ }^{75}$ Se-L-selenomethionine. J Nucl Med 6, Suppl. 6, 7-30.

30. Dangin M, Boire Y, Guillet C, et al. (2002) Influence of the protein digestion on protein turnover in young and elderly subjects. J Nutr 132, 3228S-3233S.

31. Swanson CA, Patterson BH, Levander OA, et al. (1991) Human ( $74 \mathrm{Se}$ )selenomethionine metabolism: a kinetic model. Am J Clin Nutr 54, 917-926. 